\title{
Direction générale de la Santé (DGS) (2018) Agressions collectives par armes de guerre. Conduites à tenir pour les professionnels de santé*
}

\author{
B. Riou \\ (C) SFMU et Lavoisier SAS 2018
}

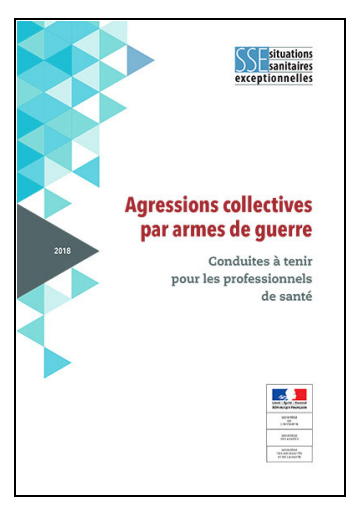

(Pr Benoît Vallet), cet ouvrage médical est publié sous l'égide de trois ministères (ministère des Solidarités et de la Santé, ministère des Armées, ministère de l'Intérieur) affirmant ainsi la collaboration interministérielle dans le domaine majeur de la prise en charge des blessés par armes de guerre lors d'attentats. Ce vade-mecum a été remis à la ministre Agnès Buzyn le 13 juillet 2018 par les Prs Pierre Carli (président du CNUH) et François Pons (ancien directeur de l'École du Val-de-Grâce du service de santé des armées) qui en ont assuré la coordination scientifique et immédiatement mis en ligne en accès libre pour les professionnels de santé (http://solidarites-sante.gouv.fr/IMG/pdf/Agressions-collectives.pdf).

Vous y trouverez des chapitres essentiels comme l'organisation des secours et des soins médicaux, l'aide médicale d'urgence dans les territoires, les spécificités des armes de guerre, en particulier lors d'agressions collectives, les principes du damage control préhospitalier et chirurgical et ses spécificités pédiatriques. La gestion hospitalière y tient une place importante avec l'accueil hospitalier, la prise en charge périopératoire, la stratégie transfusionnelle, l'organisation et la logistique hospitalières. La prise en charge médicopsychologique des victimes fait l'objet d'un chapitre, ainsi que des sujets plus rarement traités comme les traumatismes sonores. La globalité de l'approche de cet ouvrage est illustrée par le chapitre sur le parcours de soins, la réhabilitation des victimes et les aspects médicolégaux, dont l'importance pour les proches a été mise en exergue lors des attentats du 13 novembre [2]. Il faut saluer enfin le chapitre original sur la place du médecin généraliste et des autres professionnels de santé et celui sur la formation pour le grand public.

Prenez-en connaissance et n'hésitez pas le diffuser !

Liens d'intérêts : l'auteur déclare ne pas avoir de liens d'intérêts.

\section{Références}

1. Hirsch M, Carli P, Nizard R, et al (2015) The medical response to multisite terrorist attacks in Paris. Lancet 368:2535-8

2. Carli P, Pons F, Levraut J, et al (2017) The French emergency medical services after the Paris and Nice terrorist attacks: what have we learnt? Lancet 390:2735-8
B. Riou $(\bowtie)$

UMRS, Inserm 1167, IHU ICAN, service d'accueil des urgences, groupe hospitalier Pitié-Salpêtrière-Charles-Foix,

Assistance publique-Hôpitaux de Paris (AP-HP),

Sorbonne Université, F-75013 Paris, France

e-mail : bruno.riou@aphp.fr

* Direction générale de la Santé (DGS) (2018) Agressions collectives par armes de guerre. Conduites à tenir pour les professionnels de santé. Délégation à l'information et à la communication (Dicom) Éditions, Paris. Accessible : http://solidarites-sante.gouv. fr/IMG/pdf/Agressionscollectives.pdf 